\title{
Comprehensive map and functional annotation of the Mouse White Adipose Tissue Proteome
}

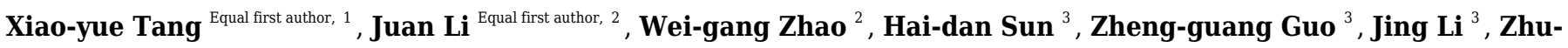 \\ fang Shen ${ }^{4}$, Tao Yuan ${ }^{2}$, Shuai-nan Liu ${ }^{4}$, Quan Liu ${ }^{4}$, Yong Fu ${ }^{2}$, Wei Sun ${ }^{\text {Corresp. } 1}$ \\ ${ }^{1}$ Core Facility of Instrument, Institute of Basic Medical Sciences, Chinese Academy of Medical Sciences/School of Basic Medicine, Beijing, China \\ 2 Department of Endocrinology, Key Laboratory of Endocrinology of Ministry of Health, Peking Union Medical College Hospital, Chinese Academy of \\ Medical Science and Peking Union Medical College, Beijing, China \\ ${ }^{3}$ Core Facility of Instrument, Institute of Basic Medical Sciences, Chinese Academy of Medical Sciences/School of Basic Medicine, Peking Union Medical \\ College, Beijing, China \\ 4 State Key Laboratory of Bioactive Substances and Functions of Natural Medicines, Institute of Materia Medica, Chinese Academy of Medical Sciences and \\ Peking Union Medical College, Diabetes Research Center of Chinese Academy of Medical Sciences, Beijing, China \\ Corresponding Author: Wei Sun \\ Email address: sunwei@ibms.pumc.edu.cn
}

White adipose tissue (WAT) plays a significant role in energy metabolism and the obesity epidemic. In this study, we sought to (1) profile the mouse WAT proteome with advanced 2DLC/MS/MS approach, (2) provide insight into WAT function based on protein functional annotation and (3) predict potential secreted proteins. A label-free 2DLC/MS/MS proteomic approach was used to identify the WAT proteome from female mouse WAT. A total of 6039 proteins in WAT were identified, among which 5160 were quantified (spanning a magnitude of $10^{6}$ ) using an intensity-based absolute quantification algorithm , and 3117 proteins were reported by proteomics technology for the first time in WAT. To comprehensively analyze the function of WAT, the proteins were divided into three quantiles based on abundance and we found that proteins of different abundance performed different functions. High-abundance proteins (the top 90\%, 1219 proteins) were involved in energy metabolism; middle-abundance proteins (90\%-99\%, 2273 proteins) were involved in the regulation of protein synthesis; and low-abundance proteins (99\%-100\%, 1668 proteins) were associated with lipid metabolism and WAT beiging. Furthermore, 800 proteins were predicted by SignalP4.0 to have signal peptides, 265 proteins had never been reported, and 5 have been reported as adipokines. The above results provide a large dataset of the normal mouse WAT proteome, which might be useful for WAT function research. 
1 Comprehensive Map and Functional Annotation of the Mouse White

3 Xiao-Yue Tang ${ }^{l \#}$, Juan $\mathrm{Li}^{2 \#}$, Wei-Gang Zhao ${ }^{2 *}$, Hai-Dan $\mathrm{Sun}^{l}$, Zheng-Guang Guo ${ }^{l}$, Jing $\mathrm{Li}^{1}$, Zhu-Fang Shen ${ }^{3}$,

4 Tao Yuan ${ }^{2}$, Shuai-Nan Liu ${ }^{3}$, Quan $\mathrm{Liu}^{3}$, Yong Fu², Wei Sun ${ }^{1 *}$

$5{ }^{1}$ Core Facility of Instrument, Institute of Basic Medical Sciences, Chinese Academy of Medical

6 Sciences/School of Basic Medicine, Peking Union Medical College, Beijing, China

$7 \quad{ }^{2}$ Department of Endocrinology, Key Laboratory of Endocrinology of Ministry of Health, Peking

8 Union Medical College Hospital, Chinese Academy of Medical Science and Peking Union Medical

9 College, Beijing, China

103 State Key Laboratory of Bioactive Substances and Functions of Natural Medicines, Institute of

11 Materia Medica, Chinese Academy of Medical Sciences and Peking Union Medical College,

12 Diabetes Research Center of Chinese Academy of Medical Sciences, Beijing, China

13 \# Contributed equally to this work

$14 *$ Corresponding authors

15 *xiehezhaoweigang@163.com (WGZ); sunwei@ibms.pumc.edu.cn (WS) 
16 Abstract

17 White adipose tissue (WAT) plays a significant role in energy metabolism and the obesity epidemic. In this study, we sought to (1) profile the mouse WAT proteome with advanced 2DLC/MS/MS approach, (2) provide insight into WAT function based on protein functional annotation and (3) predict potential secreted proteins. A label-free 2DLC/MS/MS proteomic approach was used to identify the WAT proteome from female mouse WAT. A total of 6039 proteins in WAT were identified, among which 5160 were quantified (spanning a magnitude of $10^{6}$ ) using an intensity-based absolute quantification algorithm, and 3117 proteins were reported by proteomics technology for the first time in WAT. To comprehensively analyze the function of WAT, the proteins were divided into three quantiles based on abundance and we found that proteins of different abundance performed different functions. High-abundance proteins (the top 90\%, 1219 proteins) were involved in energy metabolism; middle-abundance proteins (90\%-99\%, 2273 proteins) were involved in the regulation of protein synthesis; and low-abundance proteins (99\%-100\%, 1668 proteins) were associated with lipid metabolism and WAT beiging. Furthermore, 800 proteins were predicted by SignalP4.0 to have signal peptides, 265 proteins had never been reported, and 5 have been reported as adipokines. The above results provide a large dataset of the normal mouse WAT proteome, which might be useful for WAT function research. 


\section{Introduction}

36 Mammals have two types of adipose tissue, brown adipose tissue (BAT) and white adipose tissue (WAT). BAT dissipates energy and increases fatty acid oxidation and heat production by uncoupled respiration, which is mediated by uncoupling protein-1 (UCP1) $[1,2]$. The primary function of WAT is to store energy in lipid droplets in the form of triacylglycerols (TAGs) [3]. WAT also secrete hormones and cytokines to regulate energy intake, energy expenditure, and carbohydrate and lipid metabolism, e.g., adiponectin, leptin, and plasminogen activator inhibitor1 [4]. Furthermore, many studies have found that these hormones and cytokines are associated with diseases. For example, adiponectin (ADI), one of the most abundant adipokines produced by adipocytes, plays a fundamental role in maintaining vascular homeostasis, protecting from vascular injury and atherogenesis [5,6]. These proteins are considered a valuable resource in disease research. Therefore, a comprehensive profile of the WAT proteome will provide a basic understanding of WAT function and benefit disease studies.

The earliest study of the normal WAT proteome was from Sanchez et al. in 2001 [7]. In this study, 61 protein entries from mouse WAT were identified from one hundred and nine spots in a 2D-PAGE gel. Later, to optimize the resolution of WAT proteins, Lanne et al. used thiourea to obtain distinct spots in a 2D gel and finally identified 108 proteins from 140 spots in a 2D gel taken from the WAT of the epididymal fat pads of obese, diabetic ob/ob mice [8]. In 2013, ChengHsien et al. analyzed proteins differentially expressed in the WAT of mice fed diets with or without bitter melon seed oi for 11 weeks using two dimensional gel electrophoresis and finally identified 23 proteins from 30 spots[9]. With the development of mass spectrometry technology, more proteins can be identified in WAT. In 2007, Adachi et al. [10] investigated the adipocyte proteome by combining one-dimensional gel electrophoresis and on-line electrospray tandem mass spectrometry with biochemical procedures for the subfractionation of the 3T3-L1 adipocyte cellular proteome (nuclei, mitochondria, membrane, and cytosol). Finally, they reported 3287 SwissProt annotated proteins in 3T3-L1 adipocytes. In 2009, Forner et al. [11] analyzed the mouse fat mitochondria proteome response to cold-induced thermogenesis. By combining HPLC and 
62

high-resolution quantitative linear ion trap (LTQ)-Orbitrap mass spectrometry with the SILAC method, they identified 2434 proteins from the mitochondria of mouse adipose tissue. In 2010, Xie et al. [12] identified 1493 adipocyte proteins isolated from abdominal adipose tissue using a onedimensional SDS-PAGE and HPLC-ESI-MS/MS approach. In 2018, Peter et al. [13] analyzed mesenteric, omental, and uterine adipose tissue groups from the peritoneal cavities of young and aged C57BL/6J mouse cohorts with SDSPAGE gel combination with LC-MS/MS method. They finally identified 2308 protein groups and quantified 2167 groups. There were also some studies to investigate differently expressed proteins in adipose tissue to unveil the mechanisms underlying adipose tissue dysfunction and understand the functional mechanism of WAT[14-17].

Presently, due to its high-throughput and high-sensitivity capacities, proteomics technology has garnered a lot of attention recently in biological research. The number of identified gene products in two proteome studies of human tissues and body fluids even approached the number of protein-coding genes in the complete human genome [18-20].

Therefore, in this study, we sought to (1) profile the mouse WAT proteome with advanced 2DLC/MS/MS approach, (2) provide insight into WAT function based on protein functional annotation and (3) predict potential secreted proteins. In previous reports of WAT proteome, SDSPAGE was the most commonly used method for protein separation, but its peak capacity was limit. 2DLC approach could greatly improve the peak capacity by the orthogonality of the twodimensional separations, therefore, it could identify more proteins [21-23]. Thus, 2DLC approach was used in our study. For mass spectrometer, Triple TOF 5600 with higher sensitivity and resolution was used in the present study. To this end, we performed an in-depth WAT proteomic analysis using normal mouse WAT samples. Qualitative and quantitative analysis were applied to comprehensively profile the mouse WAT proteome. The function of WAT and the potentially secreted protein prediction was annotated with bioinformatic analysis. Our data provides a comprehensive mouse WAT proteome dataset, which might be useful for WAT function research. 
88

89

90

91

92

93

\section{Experimental procedures}

\section{Animals}

Six- to eight-week-old female C57BL/6J mice were purchased from HFK Bioscience Laboratories (Beijing, China). All mice were maintained with free access to water and food and maintained under SPF conditions and a $12 \mathrm{~h} \mathrm{light/dark} \mathrm{cycle} \mathrm{at} 23 \pm 2{ }^{\circ} \mathrm{C}$. The mice were fed with a standard chow (SC; 10\% lipids) diet for 22 weeks. All animals were handled according to the Standards for Laboratory Animals (GB14925-2001) and the Guideline on the Humane Treatment of Laboratory Animals (MOST 2006a) established by the People's Republic of China. The two guidelines were conducted in adherence to the regulations of Institutional Animal Care and Use Committee (IACUC) and all animal procedures were approved by IACUC (approval number: SCXKBeijing-2009-0004). All efforts were made to minimize suffering.

\section{Preparation of WAT samples}

A total of $6 \mathrm{C} 57 \mathrm{BL} / 6 \mathrm{~J}$ mice was applied to collect $300 \mathrm{mg}$ gonadal WAT. Phosphate buffer solution (PBS) was used to wash the tissue. The tissues were gently agitated by vortexing, and centrifuged at $1000 \mathrm{~g}$ for five minutes. After the solution was removed. $1 \mathrm{~mL}$ lysis buffer (protein extraction kit, P1250-50, Applygen, Beijing) and $1 \mu \mathrm{L}$ protease inhibitor cocktail (50x, P1265-0.5, Applygen, Beijing) (1:1000) were added to the tissue. And the tissue was homogenized using a spinning blade tissue homogenizer (IKA R104, Janke \& Kunkel KG.IKA-werk, Germany) on ice for complete lysis. $2 \mathrm{~mL}$ protein extraction regent were added to the tissue and kept in $4{ }^{\circ} \mathrm{C}$ for 40 minutes to separate lipid completely. After centrifugation at $4^{\circ} \mathrm{C}$ for 10 minutes, the solution is divided into two phases and the middle of the two phases is protein membrane. The upper and lower two layers of liquid are removed and the protein membrane is attached to the centrifugal tube wall. Protein dissolution was performed by adding a buffer solution containing $7 \mathrm{M}$ urea, 2 M thiourea, $65 \mathrm{mM}$ DTE, and $83 \mathrm{mM}$ Tris (Sigma-Aldrich, St. Louis, MO, USA). After $10 \mathrm{~min}$ of $20,000 \times \mathrm{g}$ centrifugation at $4^{\circ} \mathrm{C}$, the supernatants were collected and stored at $-80^{\circ} \mathrm{C}$. The Bradford assay with Bradford reagents (Thermo Fischer Scientific, USA) was supplied to measure the protein concentration of the WAT samples. The six WAT protein samples were pooled into one 
115

116

117

118

119

120

121

122

123

124

125

126

127

128

129

130

131

132

133

134

135

136

137

138

139

140

141

mixed sample with the same protein amount. The WAT proteins were prepared using filter-aided sample preparation methods [24]. The sample (100 $\mu \mathrm{g}$ in total) was deoxidized with $20 \mathrm{mM}$ DTT and alkylated with $50 \mathrm{mM}$ IAA. Then, the WAT proteins were loaded onto a $10 \mathrm{kDa}$ filter device (micro Biospin, Nanosep 10K Omega; PALL, USA) and centrifuged at $14,000 \mathrm{~g}$ at $4^{\circ} \mathrm{C}$. After washing twice with UA $(8 \mathrm{~mol} / \mathrm{L}$ urea in $0.1 \mathrm{~mol} / \mathrm{L}$ Tris-HCl, $\mathrm{pH} 8.5)$ and four times with 25 $\mathrm{mmol} / \mathrm{L} \mathrm{NH}_{4} \mathrm{HCO}_{3}$, the samples were digested with trypsin (Trypsin Gold, mass spec grade, Promega, WI, USA, enzyme to protein ratio of 1:50) and incubated at $37^{\circ} \mathrm{C}$ overnight. After digestion, HLB 3 cc extraction cartridges (Oasis, Waters, Ireland) were applied to desalt the peptides. $500 \mu \mathrm{l} 0.1 \%$ formic acid cleaned the peptide three times and peptide was finally eluded with $500 \mu \mathrm{L} 100 \%$ ACN. The pooled peptide elution was vacuum dried and stored at $-80^{\circ} \mathrm{C}$.

\section{LC-MS/MS}

The pooled peptide mixture was first fractioned using a high-pH RPLC column from Waters (4.6 $\left.\mathrm{mm} \times 250 \mathrm{~mm}, \mathrm{C}_{18}, 3 \mu \mathrm{m}\right)$. The sample was loaded onto the column in buffer $\mathrm{A} 1(\mathrm{pH}=10)$. Peptides were separated and eluted from 5\% B1 to 90\% B1 (90\% ACN; pH = 10, flow rate, 1 $\mathrm{mL} / \mathrm{min}$ ) in $60 \mathrm{~min}$. The eluted peptides were collected at a rate of one fraction per minute and we concatenated the 60 collected fractions into20 fractions (concatenation scheme: $1+21+41,2+$ $22+42$, etc.). Each pooled sample was analyzed on a RP-C ${ }_{18}$ self-packing LC column $(75 \mu \mathrm{m} \times 100$ $\mathrm{mm}, 3 \mu \mathrm{m})$. The eluted gradient was $5-30 \%$ buffer B2 (0.1\% formic acid, $99.9 \% \mathrm{ACN}$; flow rate, $0.5 \mu \mathrm{L} / \mathrm{min}$ ) for $60 \mathrm{~min}$.

The eluted peptide was measured using LC-MS instrumentation consisting of a nano source to TripleTOF 5600 mass spectrometer. High sensitivity mode and the following parameters were used when acquiring the MS/MS data. MS data were acquired with 30 data-dependent MS/MS scans per every full scan and full scans were acquired at a resolution of 40,000 and MS/MS scans at 20,000. 35\% normalized collision energy and charge state screening (including precursors with +2 to +4 charge state) was applied. MS/MS scans were performed at the scan range of m/z 1001800 with the scan time of $100 \mathrm{~ms}$. Dynamic exclusion was set to $15 \mathrm{~s}$ to avoid repeated sequencing of identical peptides. 
142

143

144

145

146

147

148

149

150

151

152

153

154

155

156

157

158

159

160

161

162

163

164

165

166

167

168

\section{Data processing}

The MS/MS spectra were processed using Mascot software (version 2.3.02, Matrix Science, London, UK) and searched against the SwissProt mouse database from the UniProt website (http://www.UniProt.org). The specificity of trypsin digestion was set for cleavage after K or R and two missed cleavage sites in the trypsin digestion were allowed. The fixed modification was carbamidomethylation of cysteine in Mascot. Deamidation of asparagine and glutamine and the oxidation of methionine were specified as variable modifications. The parent ion tolerance was set to $10 \mathrm{ppm}$ and the fragment ion mass tolerance was set to $0.05 \mathrm{Da}$. Mascot search results were further validated using Scaffold (version 4.3.2, Proteome Software Inc., Portland, OR). Peptide identifications were accepted at an FDR less than $1.0 \%$ by the Scaffold Local FDR algorithm and were determined by searching a reverse database. Proteins that contained similar peptides and could not be differentiated based on MS/MS analysis alone were grouped to satisfy the principles of parsimony. Proteins sharing significant peptide evidence were grouped into clusters. Protein identifications were accepted if they could achieve a false discovery rate (FDR) of less than 1.0\% and contain at least 2 uniquely identified peptides.

To rank the relative abundance of proteins, an intensity-based absolute quantification (iBAQ) algorithm was used [25]. The detailed protocol was provided below:

(1) The protein intensities were first computed by Progenesis LC-MS (v2.6, Nonlinear Dynamics, UK) as the sum of all identified peptide intensities (maximum peak intensities of the peptide elution profile, including all peaks in the isotope cluster).

(2) The protein intensities were then divided by the number of theoretically observable peptides (calculated by in silico protein digestion; all fully tryptic peptides between 6 and 30 amino acids were counted). The resulting intensities were iBAQ values. which are shown as "Absolute iBAQ intensities".

(3) The relative iBAQ intensities were computed by dividing the absolute iBAQ intensities by the sum of all absolute iBAQ intensities.

(4) The relative iBAQ intensities were applied to estimate the estimated protein abundances 
169 (the proportions of protein amounts to total WAT protein amount). The estimated protein 170 abundances in WAT were finally calculated by multiplying the relative iBAQ intensities by 171 theoretically relative molecular mass.

172 (5) The percentage was computed by dividing the estimated protein abundances by the sum of 173 all estimated protein abundances.

174 5. Functional annotation

175 Gene Ontology (GO) was performed using the PANTHER database (Protein Analysis through 176 Evolutionary Relationships, http://www.pantherdb.org/). Proteins were divided into cellular 177 components, biological processes, and molecular functions based on their functional annotations. 178 When more than one assignment was available, all the functional annotations were considered in 179 the results.

180 The Ingenuity Pathway Analysis (IPA, Ingenuity Systems, Mountain View, CA) database was 181 used to analyze canonical pathways. The SwissProt accession numbers of the identified proteins 182 were uploaded into the IPA using default settings. This analysis was focused on the pathways and 183 functions in which the proteins are expected to be involved. All identified WAT proteins, high, 184 middle and low-abundance proteins were analyzed respectively.

All proteins were used for potential secreted protein prediction. For this purpose, the SwissProt 186 accession numbers were input into SignalP 4.1 (http://www.cbs.dtu.dk/services/SignalP/) [17]. This software was used to predict the N-terminal signal peptides for classically secreted proteins. 188 


\section{Results}

\section{Workflow of proteome analysis}

In this study, samples of WAT from six C57BL/6J female mice were used to generate a comprehensive profile of the normal mouse WAT proteome. The extracted proteins were digested using the filter-aided sample preparation (FASP) method [24]. The digested peptides were first separated by high-pH RPLC and mixed into 20 fractions and analyzed by nanoRPLC-MS/MS in three replicates. The peak intensity-based semiquantification method iBAQ was used to estimate the abundance of the quantified proteins. The PANTHER classification system and IPA were applied to annotate the function of WAT. The SignalP 4.1 Server was applied to predict secreted proteins (Supplemental Fig.S1).

\section{A comprehensive profile of the WAT proteome}

In this study, pooled WAT samples were used to establish a large database for the WAT proteome. All of 516,703 spectra, 60,215 unique peptides and 6,039 proteins were identified (FDR $<1 \%$ at the protein and peptide level with at least two unique peptides for each protein) (Detailed data provided in Tables S1 - S3). Among the 6039 identified proteins, 77\% had molecular weight between 10kD-90kD (Supplemental Fig.S2). 5057 proteins (84\%) of the 6039 proteins were identified in the three replicates (Fig. 1A).

In order to improve our understanding of the WAT proteome and facilitate the development of WAT functional annotation, quantitation of WAT proteins was performed. Among the 6039 identified proteins, 5270 proteins could be quantified by the peak intensity-based semiquantification method iBAQ [25] (Fig. 1B). As for the correlation of protein quantification, the correlation of two replicates was approximately 0.98 (Supplemental Fig.S3), indicating good LC/MS/MS reproducibility. The proteins with technical CVs greater than 0.3 were excluded from further analysis in order to reduce the interference of technical variation, and a total of 5160 WAT proteins (Fig. 1C, Table S4) were kept. The relative protein abundance spans 6 orders of magnitude. We divided the quantified proteins into three quantiles based on protein abundance: high- (the top 90\%, 1219 proteins), middle- (90\%-99\%, 2273 proteins) and low-abundance proteins (99\%-100\%, 1668 proteins). Additionally, Table 1 listed top ten abundant proteins 
217 quantified with the iBAQ algorithm in our research. The ten proteins account for $20 \%$ of the total 218 WAT protein abundance. Carbonic anhydrase 3, vimentin and polymerase I and transcript release 219 factors are associated with fatty acid metabolism, adipogenesis and lipolysis, respectively [26-28]. 220 Galectin-1 (GAL1) has been proposed as an early marker of adipocyte differentiation and to be

221

222

223

224

225

226

227

228

229

230

231

232

233

234

235

236

237

238

239

240

241 actively involved in adipose tissue development [29, 30]. The expression of transgelin was associated with the development of white adipocyte, and thought to begin with progenitor cells from the perivascular compartment (pericytes) in WAT [31]. Other three proteins, tropomyosin (beta chain), desmin and myosin light polypeptide 6, were cytoskeletal proteins.

When comparing with previous studies (Table 2, Fig.2A), approximately 58\% of the fat mitochondria proteome, $60 \%$ of the mesenteric, omental, and uterine adipose tissue proteome, $63 \%$ of the 3T3-L1 adipocyte proteome and $92 \%$ of the adipocyte proteome (isolated from abdominal adipose tissue) was overlapped with our study, respectively. When all data from these four studies were combined, 352 proteins were detected in all of the studies and 3117 proteins from our results were reported for the first time by proteomics technology in WAT. Among common proteins (Fig.2B), high- and middle-abundance proteins contributed about 84\%. And among specific proteins, less $(7 \%)$ high-abundance proteins were found in our study. When comparing previous study with our high-abundance proteins (Supplemental Fig.S4), 177 proteins were firstly reported in our study and 85\% (1042 proteins) of our high-abundance proteins could be identified previously. The above data showed that most high abundance proteins $(85 \%)$ and middle abundance proteins $(52 \%)$ were identified by previous reports, and less proteins low abundant proteins $(26 \%)$. The possible reasons might come from the proteome approach. Due to the limitations of separation method and mass spectrometer in previous studies, most identified proteins in previous reports were high or medium ones, therefore, these proteins had more chances to be identified in our study.

\section{The functional annotation of the WAT proteome}


242

243

244

245

246

247

248

249

250

251

252

253

254

255

256

257

258

259

260

261

262

263

264

265

266

267

268

To explore the function of WAT more comprehensively, PANTHER classification system (http://www.pantherdb.org/genes/batchIdSearch.jsp) and IPA tool (http://www.ingenuity.com/) were applied to the functional classification and pathway analysis.

The identified proteins were functionally categorized using Gene Ontology annotation terms. As shown in Fig. 3A, nearly $85 \%$ of the proteins were present in cells, including cell part (40.6\%, including intracellular part and endomembrane system), organelles (26.3\%, including intracellular organelle and non-membrane bounded organelle) and macromolecular complex $(17.4 \%$, including membrane protein complex, catalytic complex and ribonucleoprotein complex). The major molecular functions of WAT proteins were catalytic activity (44\%, including hydrolase activity and transferase activity) and binding (37.5\%, including protein binding and heterocyclic compound biding). High-abundance proteins were enriched with antioxidant activity (5.9\%) and structural molecular activity (10.1\%) when compared with the middle and low-abundance proteins (Fig. 3B). Using iBAQ analysis, the high-abundance proteins with antioxidant and catalytic activity accounted for $35 \%$ of all the high-abundance proteins, and the abundance of proteins with structural molecular activity accounted for 8\%. For biological processes (Fig. 3C), approximately half of the WAT proteins were associated with metabolic processes $(25.1 \%)$ and cellular processes (31\%). High-abundance proteins were enriched with cellular component organization or biogenesis (11.3\%), multicellular organismal process (4.5\%) and biological adhesion functions $(1.7 \%)$. The above results show that energy metabolism is an important function of WAT.

To identify the major biological pathways involved with the identified proteins, IPA was used for canonical pathway enrichment analysis (Fig. 3D, Supplemental Fig. S5 and Table S5). The functions of WAT include protein synthesis (such as the protein ubiquitination pathway, EIF2 signaling, mTOR signaling, and the regulation of eIF4 and p70S6K signaling), energy metabolism (such as mitochondrial dysfunction, sirtuin signaling, PI3K/AKT signaling, and oxidative phosphorylation), adipogenesis (such as PI3K/AKT signaling) and the regulation of insulin action (such as integrin signaling). The function of the high-abundance proteins was mainly related to energy metabolism (mitochondrial dysfunction, oxidative phosphorylation, and sirtuin signaling). 
269 The primary function of the middle-abundance proteins was protein synthesis (mTOR signaling, 270 regulation of eIF4 and p70S6K signaling, EIF2 signaling and tRNA charging). The functions of

271 low-abundance proteins were WAT beiging (NGF and IL-4 signaling) and lipid metabolism (super 272 pathway of inositol phosphate compounds, 3-phosphoinositide degradation and 3273 phosphoinositide biosynthesis).

\section{4. The analysis of WAT secreted proteins}

275 Endocrine function is one of the key roles of adipocytes, and adipose tissue can synthesis several hormones, called adipokines or adipocytokines. We applied the SignalP 4.1 Server to predict potential secreted proteins. In all, 800 proteins were found to have signal peptides (Table S6), of which 200 proteins belong to the high-abundance proteins, 312 belong to the middleabundance proteins and 183 belong to the low-abundance proteins. The abundance of all the proteins with signal peptides was $15.6 \%$ in the WAT proteome. In 2018, Ali et al. identified 453 proteins from adipocytes that had previously been reported to be secreted proteins [32], of which 265 proteins were found in our results. Moreover, 5 WAT proteins were confirmed as adipokines: resistan, adiponectin, apolipoprotein E, haptoglobin and angiotensinogen [33-35].

In addition, the peak intensity-based semiquantification method iBAQ was applied to estimate the abundance of the identified 800 proteins, of which 694 proteins could be quantified [36]. The relative protein amounts span 5 orders of magnitude, from Semaphorin-4A (9.74E-01) to alpha-2HS-glycoprotein (3.81E+04) (Fig. 2C). 


\section{Discussion}

White adipose tissue(WAT) stores energy in lipid droplets in the form of TAGs[3] and secrete hormones and adipokines to regulate energy intake, energy expenditure, and carbohydrate and lipid metabolism [4]. A comprehensive profile of the WAT proteome will provide a basic understanding of WAT function and benefit disease studies. In the present study, we established a large database for the WAT proteome by advanced 2D LC-MS/MS. Total 6,039 proteins were identified, of which 5270 proteins could be quantified by the peak intensity-based semiquantification method iBAQ. Functional annotation was further explored and we found that proteins of different abundance performed different functions.

Canonical pathway enrichment analysis revealed that one of the primary functions of WAT was protein synthesis, and related significant pathways was EIF2 signaling. Global translation and apoptosis can be started by phosphorylation of EIF2-a [37] and eIF4E was released by phosphorylation of eIF4E-BP binds to 5' capped mRNAs to enhance their translation [38]. Another significant WAT pathway is the PI3K/AKT signaling pathway, which plays a significant role in phytol-enhanced adipogenesis and glucose uptake of adipocytes and lipolysis inhibition [39, 40]. These results show the synthetic metabolism function of WAT, which is consistent with the traditional energy storage role of WAT [33]. Furthermore, a previous study has suggested that adipose tissue plays an important role in insulin resistance [41]. In our result, integrin signaling performed this function. A total of 103 proteins were found to be involved in this pathway, such as Akt and ILK. ILK can interact with the integrin cytoplasmic domains, and integrins rely on ILK for signal transduction. The pseudokinase domain of ILK is an important domain for the recruitment of adaptor proteins and/or signaling molecules, such as Akt and PDK1. Integrins are important regulators of insulin action and represent novel therapeutic targets to treat the underlying insulin resistance associated with T2D [40].

The WAT proteome was divided into high, middle and low abundance proteins by iBAQ quantitative method. According to IPA functional annotation high-abundance proteins was mainly related to energy metabolism (mitochondrial dysfunction, oxidative phosphorylation, and sirtuin 
316

317

318

319

320

321

322

323

324

325

326

327

328

329

330

331

332

333

334

335

336

337

338

339

340

341

342

signaling). Sixty-five proteins were involved in sirtuin signaling, such as sirt2. During energy deficiency (caloric restriction or fasting) the expression of sirt 2 increases $[42,43]$. In this situation, adipogenesis was suppressed and adipocyte lipolysis was increased to promote fuel availability by sirt2 [42]. In another significant pathway, oxidative phosphorylation occurs in mitochondria and comprises an electron-transfer chain, which is driven by substrate oxidation and is coupled to the synthesis of ATP through an electrochemical transmembrane gradient. When energy needs increase, fatty acid oxidation supplies an important source of energy for ATP production [44]. In oxidative phosphorylation, 60\% ubiquinone oxidoreductase (including NDUFS4, NDUFA3, NDUFA4), 46\% ubiquinol cytochrome c oxidoreductase (including UQCRFS, UQCRC2) and 63\% ATP synthase (including ATP5A1, ATP5D) were high-abundance proteins (Supplemental Fig.S6A). The other function of mitochondria is adipogenesis, which is accompanied by mitochondrial biogenesis [45]. The mitochondria provides key intermediates for the synthesis of triacylglycerolmight and further plays a critical role in lipogenesis [46]. For mitochondrial dysfunction, many significant proteins were high-abundance proteins, including aconitase 2 and cytochrome c oxidase. Aconitase 2 was an enzyme to catalyze the interconversion of citrate via cisaconitase in the second step of the TCA cycle. Cytochrome c oxidase was the terminal enzyme of the mitochondrial respiratory chain and a multi-submit enzyme complex to transfer electrons in mitochondrial (Supplemental Fig.S6B). These results showed that besides basic physiological functions (structural molecular functions), high-abundance proteins also have important energy metabolism functions in WAT.

IPA analysis showed that the primary function of the middle-abundance proteins was protein synthesis (mTOR signaling, regulation of eIF4 and p70S6K signaling, EIF2 signaling and tRNA charging). According to a previous study, protein synthesis is associated with adipogenesis [47]. The concentration of numerous mitochondrial proteins such as cytochrome c had a 20 - to 30 -fold increase during differentiation of 3T3-L1 cells (a preadipocyte cell line). The expression of many nucleus-encoded mitochondrial genes during adipogenesis was also found to have statistically significant increases by mRNA profiles. The increase in mitochondrial proteins is not completely 
343

344

345

346

347

348

349

350

351

352

353

354

355

356

357

358

359

360

361

362

363

364

365

366

367

368

369

achieved by enhanced transcription but is also involved in posttranscriptional regulation. Adipose conversion (preadipocytes to adipocytes) would be accompanied by mitochondrial proliferation, which underscores the essential role of mitochondria in the key aspects of lipid metabolism [47].

The functions of low-abundance proteins were WAT beiging (NGF and IL-4 signaling) and lipid metabolism (super pathway of inositol phosphate compounds, 3-phosphoinositide degradation and 3-phosphoinositide biosynthesis). NGF signaling plays a key role in the beiging of WAT by regulating the plasticity of intra-adipose sympathetic arborizations in mouse inguinal WAT in response to a cold challenge. Intra-adipose sympathetic plasticity was suppressed by the blockage of NGF signaling, and moreover, the cold-induced beiging process of WAT was suppressed[48]. IL-4 signaling is involved in the cold-induced biogenesis of beige fat. All of 22 proteins were found to be involved in this pathway, including STAT6. STAT6 is thought to be necessary for the cold-induced remodeling of WAT into beige fat. Qiu et al. [49] found that coldinduced expression of the protein UCP1 in subcutaneous WAT decreased by $\sim 12$-fold in Stat6-/mice, accompanied by an increase in oxygen consumption. Accordingly, Stat6-/- mice maintained thermal homeostasis at a lower body temperature. Because beiging of WAT results in enhanced energy expenditure, it has increasingly gained attention for its potential application in the prevention and treatment of obesity and type 2 diabetes[50,51].

In addition, 800 proteins were found to be secreted proteins in our result, of which 694 proteins could be quantified by iBAQ. The top 5 newly identified (most abundant) secreted proteins were carboxylesterase 1D, serine protease inhibitor A3K, apolipoprotein A-I, carboxylesterase 1C and fibrillin-1.Carboxylesterase 1D, a major lipase in WAT, may mediate some or all hormonesensitive lipase -independent lipolysis in adipocytes[52]. Apolipoprotein A-I was associated with cholesterol transport in high-density lipoprotein [53]. Fibrillin-1 was related with tissue homeostasis [54]. It was reported to be up-regulated in the WAT of diet-induced obese mice [55], which was affected by the structural changes when adipose tissue enlargement. The other two proteins were serine protease inhibitor A3K and carboxylesterase 1C. Serine protease inhibitor A3K was a contrapsin to inhibit trypsin-like proteases[56, 57]. Carboxylesterase $1 \mathrm{C}$ was involved 
370 in the detoxification of xenobiotics and in the activation of ester and amide prodrugs and in the 371 extracellular metabolism of lung surfactant. No studies were reported to explore the correlation 372 between them and white adipose tissue. Their functions in WAT need more work. 


\section{Conclusion}

375 In conclusion, using high-pH reverse-phase liquid chromatography (hp-RPLC) integrated with 376 a TripleTOF 5600 mass spectrometer, we provided the largest dataset from the normal mouse 377 WAT proteome up to now. Our WAT proteome included many proteins that had not been 378 previously reported by proteomic approach. We further provided insight into WAT function based 379 on protein functional annotation and found that proteins of different abundance performed 380 different functions. Finally, potential secreted proteins prediction was performed. Our data set 381 provided a comprehensive mouse WAT proteome, which might be useful for WAT function 382 research.

383 In the future, the following issues should be addressed: With the emergence of new instruments 384 (e.g., Orbitrap Fusion Lumos Tribrid mass spectrometer), labeling reagents (e.g., Ten-plex TMT 385 labeling) and new techniques (e.g., the Data Independent Acquisition technique), high-throughput 386 proteome analysis has come to fruition and might provide a more precise quantification and 387 functional analysis. Besides, multiple sample preparation methods and adipose tissue samples from multiple regions will be needed to truly obtain a comprehensive WAT proteome. Furthermore, with the development of genomics, transcriptomes, proteomics and metabolomics, the combination of multiple omic analysis would lead to a better platform for routine clinical research. 


\section{Acknowledgments}

393 This work was supported by National Key Research and Development Program of China (No. 3942016 YFC 1306300,2018YFC0910202,), National Natural Science Foundation of China (No. $39530970650,31200614,31400669,81371515,81170665,81560121)$, Beijing Natural Science 396 Foundation (No. 7173264, 7172076), Beijing cooperative construction project (No.110651103), 397 Beijing Science Program for the Top Young (No.2015000021223TD04), Beijing Normal 398 University (No.11100704), Peking Union Medical College Hospital (No.2016-2.27), CAMS 399 Innovation Fund for Medical Sciences (2017-I2M-1-009) and Biologic Medicine Information 400 Center of China, National Scientific Data Sharing Platform for Population and Health.

\section{Conflict of interest}

402 The authors declare no conflicts of interest.

403 


\section{References}

405 1. Klingenberg, M., Uncoupling protein--a useful energy dissipator. J Bioenerg Biomembr, 1999. 31(5): p.

406 419-30.

407 2. Cannon, B. and J. Nedergaard, Brown adipose tissue: function and physiological significance. Physiol Rev, $408 \quad$ 2004. 84(1): p. 277-359.

$4093 . \quad$ Kuhnlein, R.P., Thematic review series: Lipid droplet synthesis and metabolism: from yeast to man. Lipid 410 droplet-based storage fat metabolism in Drosophila. J Lipid Res, 2012. 53(8): p. 1430-6.

4114 4cGown, C., A. Birerdinc, and Z.M. Younossi, Adipose tissue as an endocrine organ. Clin Liver Dis, 412 2014. 18(1): p. 41-58.

$4135 . \quad$ Vaiopoulos, A.G., et al., The role of adiponectin in human vascular physiology. Int J Cardiol, 2012. 155(2):

414 p. 188-93.

415 6. Nishimura, M., et al., Adiponectin prevents cerebral ischemic injury through endothelial nitric oxide 416 synthase dependent mechanisms. Circulation, 2008. 117(2): p. 216-23.

417 7. Sanchez, J.C., et al., The mouse SWISS-2D PAGE database: a tool for proteomics study of diabetes and 418 obesity. Proteomics, 2001. 1(1): p. 136-63.

419 8. Lanne, B., et al., Thiourea enhances mapping of the proteome from murine white adipose tissue.

$420 \quad$ Proteomics, 2001. 1(7): p. 819-28.

4219 9. Hsieh, C.H., et al., Altered white adipose tissue protein profile in C57BL/6J mice displaying delipidative,

422 inflammatory, and browning characteristics after bitter melon seed oil treatment. PLoS One, 2013. 8(9): p.

10. Adachi, J., et al., In-depth analysis of the adipocyte proteome by mass spectrometry and bioinformatics. Mol Cell Proteomics, 2007. 6(7): p. 1257-73.

11. Forner, F., et al., Proteome differences between brown and white fat mitochondria reveal specialized metabolic functions. Cell Metab, 2009. 10(4): p. 324-35.

12. Xie, X., et al., Characterization of the Human Adipocyte Proteome and Reproducibility of Protein Abundance by One-Dimensional Gel Electrophoresis and HPLC-ESI-MS/MS. J Proteome Res, 2010. 9(9): p. 4521-34.

13. Feist, P.E., et al., Quantitative proteomic analysis of murine white adipose tissue for peritoneal cancer metastasis. Anal Bioanal Chem, 2018. 410(5): p. 1583-1594.

14. Peinado, J.R., et al., Proteomic profiling of adipose tissue from Zmpste24-/- mice, a model of lipodystrophy and premature aging, reveals major changes in mitochondrial function and vimentin processing. Mol Cell Proteomics, 2011. 10(11): p. M111.008094.

15. Andrade, J.M., et al., Proteomic white adipose tissue analysis of obese mice fed with a high-fat diet and treated with oral angiotensin-(1-7). Peptides, 2014. 60: p. 56-62.

16. Kim, S.W., et al., Differential protein expression in white adipose tissue from obesity-prone and obesityresistant mice in response to high fat diet and anti-obesity herbal medicines. Cell Physiol Biochem, 2015. 35(4): p. 1482-98.

17. Ke, M., et al., Differential proteomic analysis of white adipose tissues from T2D KKAy mice by LC-ESIQTOF. Proteomics, 2017. 17(5).

18. Kim, M.S., et al., A draft map of the human proteome. Nature, 2014. 509(7502): p. 575-81. 
444 19. Wilhelm, M., et al., Mass-spectrometry-based draft of the human proteome. Nature, 2014. 509(7502): p. $445 \quad 582-7$.

20. Consortium, E.P., An integrated encyclopedia of DNA elements in the human genome. Nature, 2012. 489(7414): p. 57-74.

21. Gilar, M., et al., Orthogonality of separation in two-dimensional liquid chromatography. Anal Chem, 2005. 77(19): p. 6426-34.

22. Wang, Y., et al., Reversed-phase chromatography with multiple fraction concatenation strategy for proteome profiling of human MCF10A cells. Proteomics, 2011. 11(10): p. 2019-26.

23. Song, C., et al., Reversed-phase-reversed-phase liquid chromatography approach with high orthogonality for multidimensional separation of phosphopeptides. Anal Chem, 2010. 82(1): p. 53-6.

24. Wisniewski, J.R., et al., Universal sample preparation method for proteome analysis. Nat Methods, 2009. 6(5): p. 359-62.

25. Schwanhausser, B., et al., Global quantification of mammalian gene expression control. Nature, 2011. 473(7347): p. 337-42.

26. Lyons, G.E., et al., Carbonic anhydrase III, an early mesodermal marker, is expressed in embryonic mouse skeletal muscle and notochord. Development, 1991. 111(1): p. 233-44.

27. Joo, J.I., et al., Proteomic analysis for antiobesity potential of capsaicin on white adipose tissue in rats fed with a high fat diet. J Proteome Res, 2010. 9(6): p. 2977-87.

28. Aboulaich, N., et al., Polymerase I and transcript release factor regulates lipolysis via a phosphorylationdependent mechanism. Diabetes, 2011. 60(3): p. 757-65.

29. Ahmed, M., et al., Proteomic analysis of human adipose tissue after rosiglitazone treatment shows coordinated changes to promote glucose uptake. Obesity (Silver Spring), 2010. 18(1): p. 27-34.

30. Wang, P., et al., Profiling of the secreted proteins during 3T3-L1 adipocyte differentiation leads to the identification of novel adipokines. Cell Mol Life Sci, 2004. 61(18): p. 2405-17.

31. Todorcevic, M., et al., Gene expression profiles in Atlantic salmon adipose-derived stromo-vascular fraction during differentiation into adipocytes. BMC Genomics, 2010. 11: p. 39.

32. Ali Khan, A., et al., Comparative secretome analyses of primary murine white and brown adipocytes reveal novel adipokines. Molecular \&amp; Cellular Proteomics, 2018.

33. Trayhurn, P. and J.H. Beattie, Physiological role of adipose tissue: white adipose tissue as an endocrine and secretory organ. Proc Nutr Soc, 2001. 60(3): p. 329-39.

34. Trayhurn, P. and I.S. Wood, Adipokines: inflammation and the pleiotropic role of white adipose tissue. $\mathrm{Br} \mathrm{J}$ Nutr, 2004. 92(3): p. 347-55.

35. Yue, L., et al., Divergent effects of peroxisome proliferator-activated receptor gamma agonists and tumor necrosis factor alpha on adipocyte ApoE expression. J Biol Chem, 2004. 279(46): p. 47626-32.

36. Geiger, T., et al., Comparative proteomic analysis of eleven common cell lines reveals ubiquitous but varying expression of most proteins. Mol Cell Proteomics, 2012. 11(3): p. M111 014050.

37. Kimball, S.R., Eukaryotic initiation factor eIF2. Int J Biochem Cell Biol, 1999. 31(1): p. 25-9.

38. Swetha, M. and K.V.A. Ramaiah, Insulin treatment promotes tyrosine phosphorylation of PKR and inhibits polyIC induced PKR threonine phosphorylation. Arch Biochem Biophys, 2015. 585: p. 98-108.

39. Langin, D., Adipose tissue lipolysis as a metabolic pathway to define pharmacological strategies against obesity and the metabolic syndrome. Pharmacol Res, 2006. 53(6): p. 482-91. 
485

40. Williams, A.S., L. Kang, and D.H. Wasserman, The extracellular matrix and insulin resistance. Trends Endocrinol Metab, 2015. 26(7): p. 357-66.

41. Chen, X. and S. Hess, Adipose proteome analysis: focus on mediators of insulin resistance. Expert Rev Proteomics, 2008. 5(6): p. 827-39.

42. Wang, F. and Q. Tong, SIRT2 suppresses adipocyte differentiation by deacetylating FOXO1 and enhancing FOXO1's repressive interaction with PPARgamma. Mol Biol Cell, 2009. 20(3): p. 801-8.

43. Wang, F., et al., SIRT2 deacetylates FOXO3a in response to oxidative stress and caloric restriction. Aging Cell, 2007. 6(4): p. 505-514.

44. Boudina, S. and T.E. Graham, Mitochondrial function/dysfunction in white adipose tissue. Experimental Physiology, 2014. 99(9): p. 1168-1178.

45. Choo, H.J., et al., Mitochondria are impaired in the adipocytes of type 2 diabetic mice. Diabetologia, 2006. 49(4): p. 784-91.

46. De Pauw, A., et al., Mitochondrial (dys)function in adipocyte (de)differentiation and systemic metabolic alterations. Am J Pathol, 2009. 175(3): p. 927-39.

47. Wilson-Fritch, L., et al., Mitochondrial Biogenesis and Remodeling during Adipogenesis and in Response to the Insulin Sensitizer Rosiglitazone. Molecular and Cellular Biology, 2003. 23(3): p. 1085-1094.

48. Cao, Y., H. Wang, and W. Zeng, Whole-tissue 3D imaging reveals intra-adipose sympathetic plasticity regulated by NGF-TrkA signal in cold-induced beiging. Protein Cell, 2018. 9(6): p. 527-539.

49. Qiu, Y., et al., Eosinophils and type 2 cytokine signaling in macrophages orchestrate development of functional beige fat. Cell, 2014. 157(6): p. 1292-308.

50. Giordano, A., A. Frontini, and S. Cinti, Convertible visceral fat as a therapeutic target to curb obesity. Nat Rev Drug Discov, 2016. 15(6): p. 405-24.

51. Harms, M. and P. Seale, Brown and beige fat: development, function and therapeutic potential. Nat Med, 2013. 19(10): p. 1252-63.

52. Soni, K.G., et al., Carboxylesterase 3 (EC 3.1.1.1) is a major adipocyte lipase. J Biol Chem, 2004. 279(39): p. 40683-9.

53. Sackmann-Sala, L., et al., Age-related and depot-specific changes in white adipose tissue of growth hormone receptor-null mice. J Gerontol A Biol Sci Med Sci, 2014. 69(1): p. 34-43.

54. Pereira, L., et al., Pathogenetic sequence for aneurysm revealed in mice underexpressing fibrillin-1. Proc Natl Acad Sci U S A, 1999. 96(7): p. 3819-23.

55. Lopez, I.P., et al., Gene expression changes in rat white adipose tissue after a high-fat diet determined by differential display. Biochem Biophys Res Commun, 2004. 318(1): p. 234-9.

56. Takahara, H. and H. Sinohara, Mouse plasma trypsin inhibitors: inhibitory spectrum of contrapsin and alpha-1-antitrypsin. Thromb Res, 1982. 27(1): p. 45-50.

57. Takahara, H. and H. Sinohara, Inhibitory spectrum of mouse contrapsin and alpha-1-antitrypsin against mouse serine proteases. J Biochem, 1983. 93(5): p. 1411-9. 


\section{Figure legends}

524 Fig. 1 Proteome profile analysis of the normal WAT proteome.

525 (A) Venn diagram of protein identification in three technical replicates. (B) Technical variation of 526 the three replicates. (C) Quantitative protein abundance range in WAT samples with the iBAQ 527 algorithm.

528 Fig.2 Venn diagram analysis of mouse WAT proteome. (A) Venn diagram analysis of present

529 mouse WAT proteome studies. (B) Protein distribution based on abundance of WAT proteome in 530 this study.

531 Fig.3 Functional analysis of WAT proteome according to protein abundance. (A) cellular 532 components. (B) molecular function. (C) biological processes. (D) Canonical pathway of WAT 533 proteome. 


\section{Supporting Information}

535 Table S1 The qualitative results from the WAT proteome from three technical replicates.

536 Table S2 The list of peptides in the WAT proteome.

537 Table $\mathbf{S 3}$ The list of proteins in the WAT proteome.

538 Table S4 iBAQ intensity of quantified WAT proteins.

539 Table S5 IPA annotation of the WAT proteome.

540 Table S6 The list of proteins with signal peptides.

541 Supplemental Fig. S1 The mouse gonadal white adipose tissue proteome profile workflow.

542 Supplemental Fig. S2 Correlation plot of protein abundance between two runs

543 Supplemental Fig. S3 Venn diagram analysis of mouse WAT proteome with high-

544 abundance proteins in this study.

545 Supplemental Fig.S4 Top canonical pathways in WAT.

546 Supplemental Fig.S5 Mitochondrial dysfunction (A) and oxidative phosphorylation (B)

547 signaling pathway. The red-filled graph represents high-abundance protein; the gray one is 548 middle-abundance protein; and the green one is low-abundance protein). 


\section{Table 1 (on next page)}

The top ten most abundant proteins in the mouse WAT proteome. 
1 Table 1. The top ten abundant proteins in the mouse WAT proteome.

2

3

\begin{tabular}{|c|c|c|c|}
\hline Accession number & Name & iBAQ value & Percentage \\
\hline P07724 & Serum albumin & $2.87 \mathrm{E}+05$ & $9.33 \%$ \\
\hline \multirow[t]{2}{*}{ P16015 } & Carbonic anhydrase 3 & $1.21 \mathrm{E}+05$ & $1.68 \%$ \\
\hline & Polymerase I and transcript & & \\
\hline O54724 & release factor & $8.01 \mathrm{E}+04$ & $1.67 \%$ \\
\hline P58774 & Tropomyosin beta chain & $8.72 \mathrm{E}+04$ & $1.36 \%$ \\
\hline P20152 & Vimentin & $4.93 \mathrm{E}+04$ & $1.25 \%$ \\
\hline P31001 & Desmin & $4.23 \mathrm{E}+04$ & $1.07 \%$ \\
\hline Q60605 & Myosin light polypeptide 6 & $1.30 \mathrm{E}+05$ & $1.04 \%$ \\
\hline P37804 & Transgelin & $8.74 \mathrm{E}+04$ & $0.93 \%$ \\
\hline P16045 & Galectin-1 & $1.20 \mathrm{E}+05$ & $0.85 \%$ \\
\hline Q08091 & Calponin-1 & $5.05 \mathrm{E}+04$ & $0.80 \%$ \\
\hline
\end{tabular}


Table 2 (on next page)

Proteomic studies on the mouse WAT proteome. 
1 Table 2. Proteomic studies on the mouse WAT proteome.

2

3

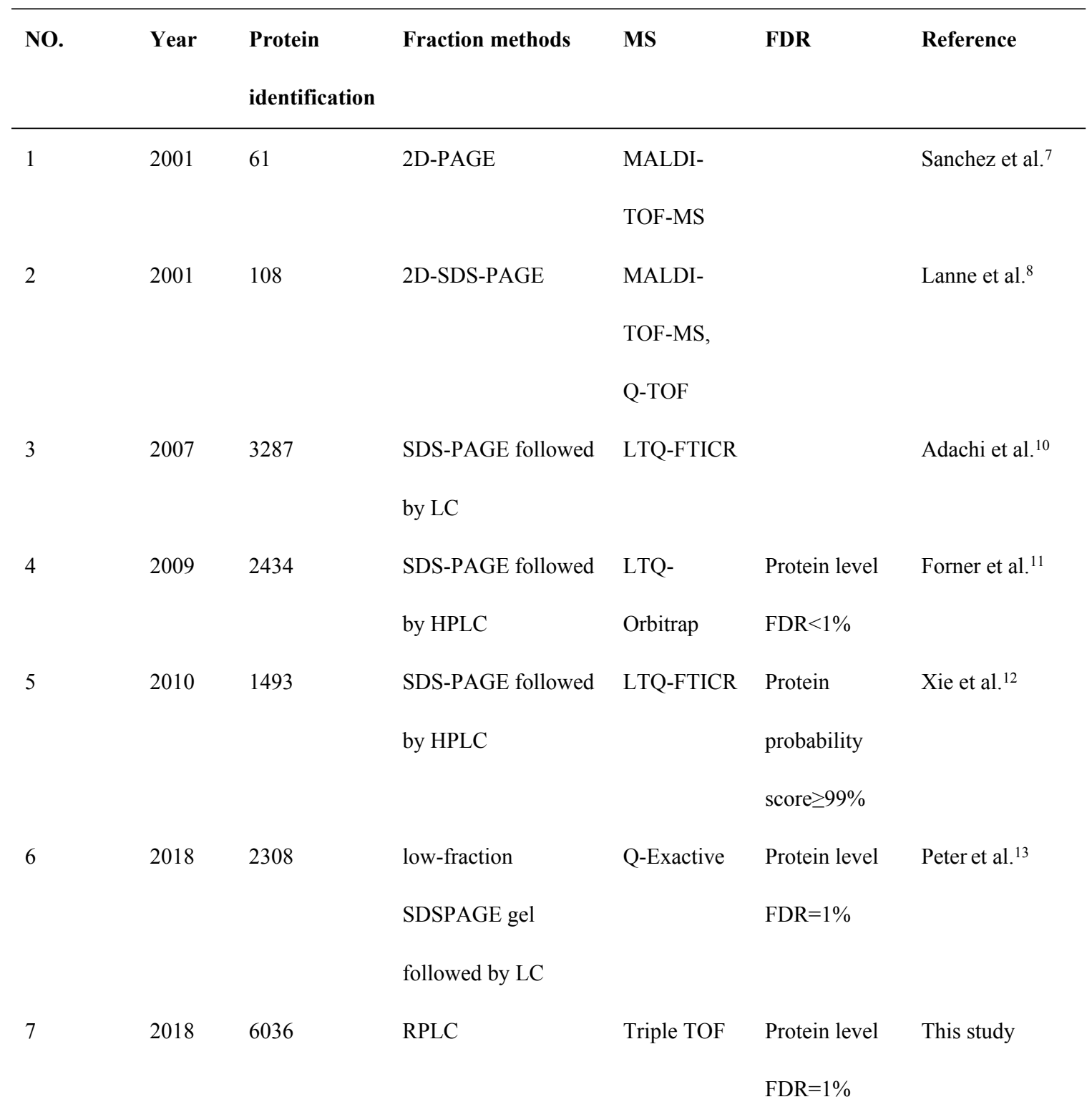

4 
Figure 1

The mouse white adipose tissue proteome profile workflow.

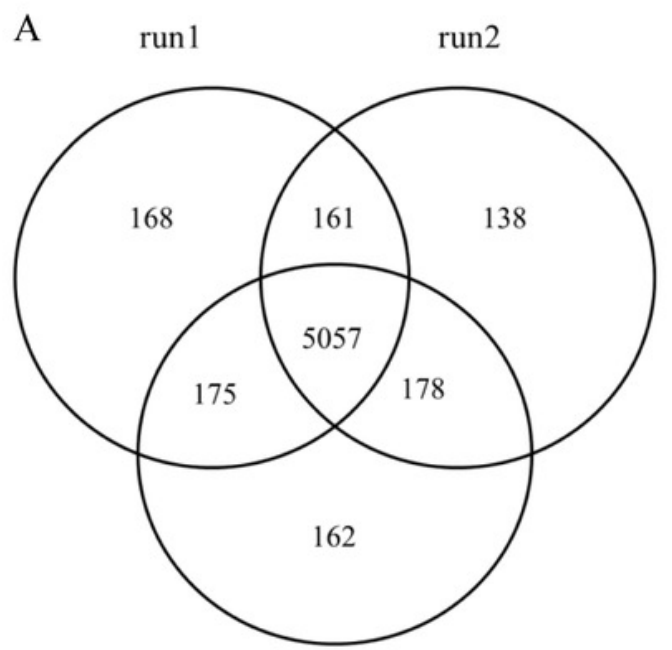

run3
B

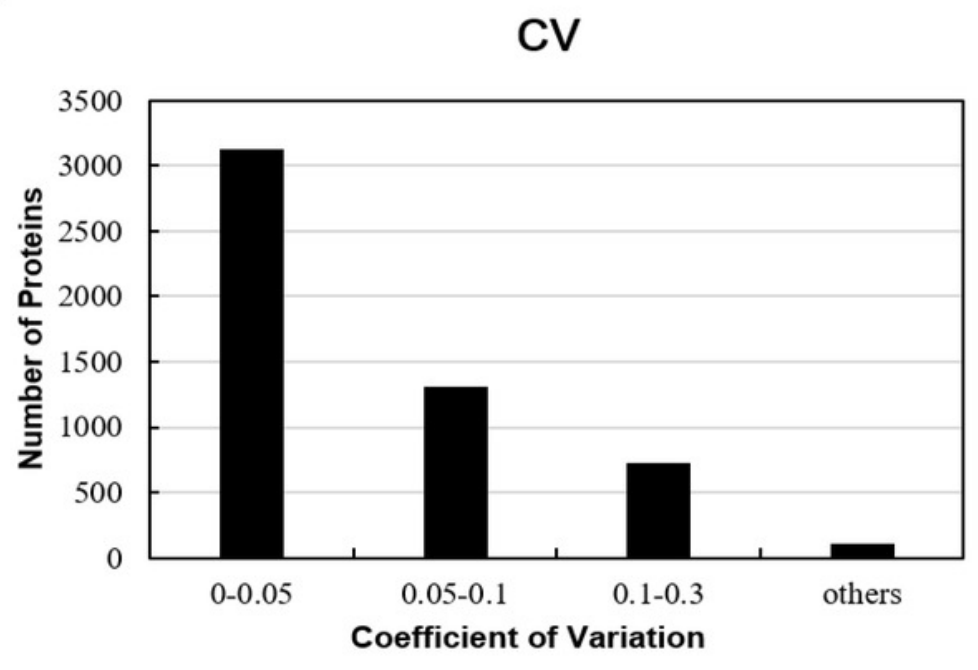

$\mathrm{C}$

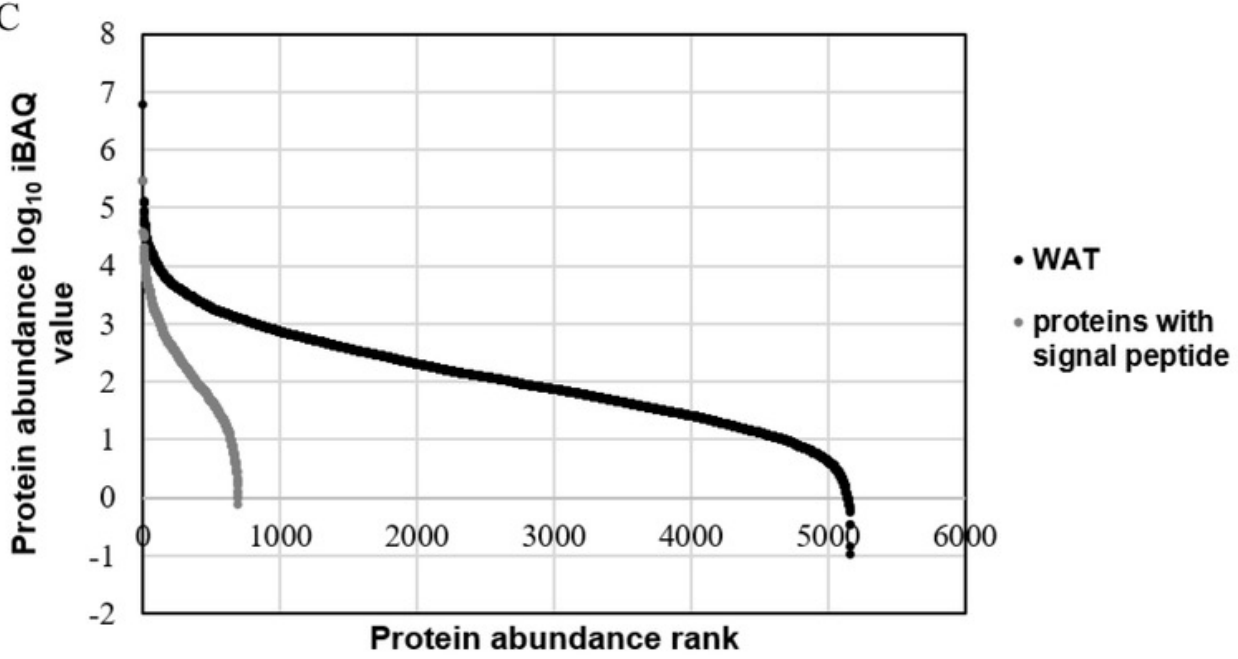




\section{Figure 2}

Proteome profile analysis of the normal WAT proteome.

(A) Venn diagram of protein identification in three technical replicates. (B) Technical variation of the three replicates. (C) Quantitative protein abundance range in WAT samples with the iBAQ algorithm. (D) Venn diagram comparing previous proteomic studies with this study.

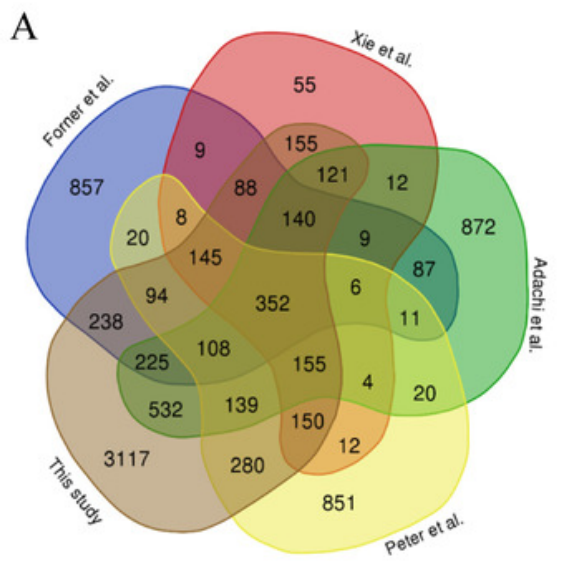

B

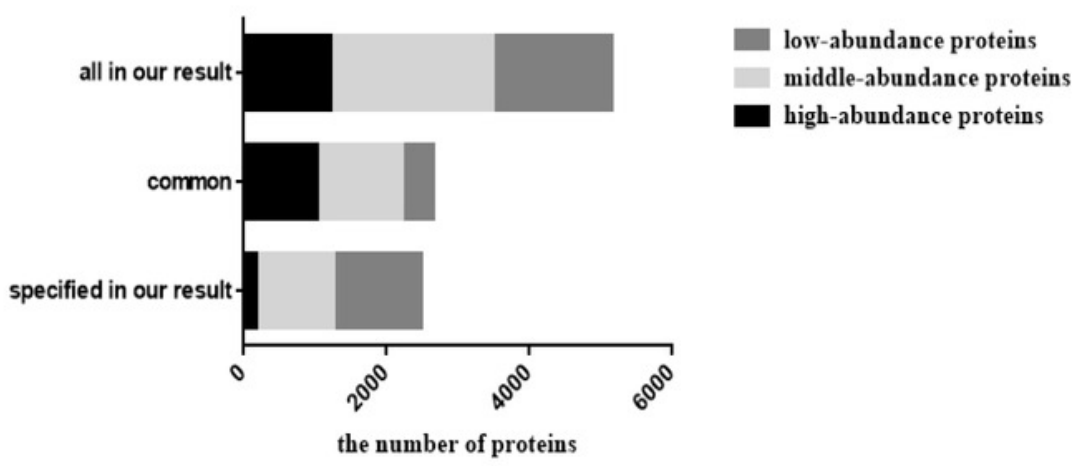


Figure 3

Functional annotation (GO and IPA analysis) of WAT proteome.

(A) cellular components, (B) molecular function, (C)biological processes, (D) top canonical pathway. 
A

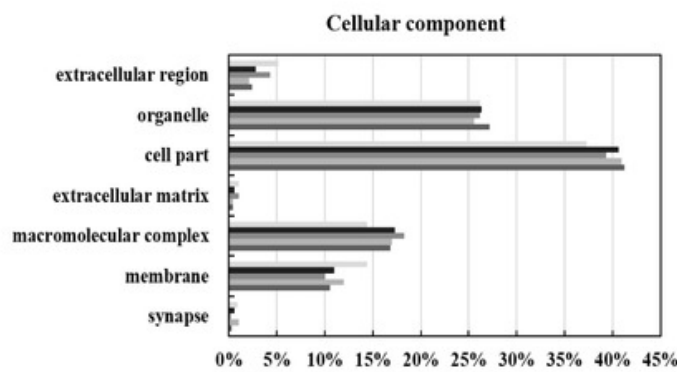

B

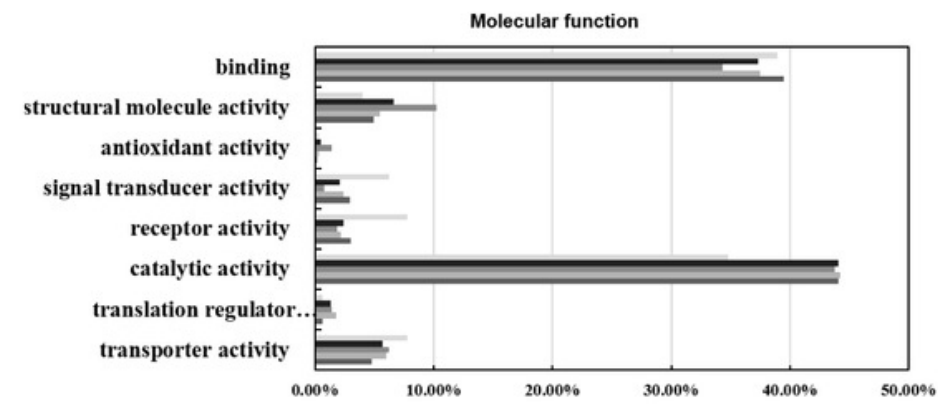

C

Biological process

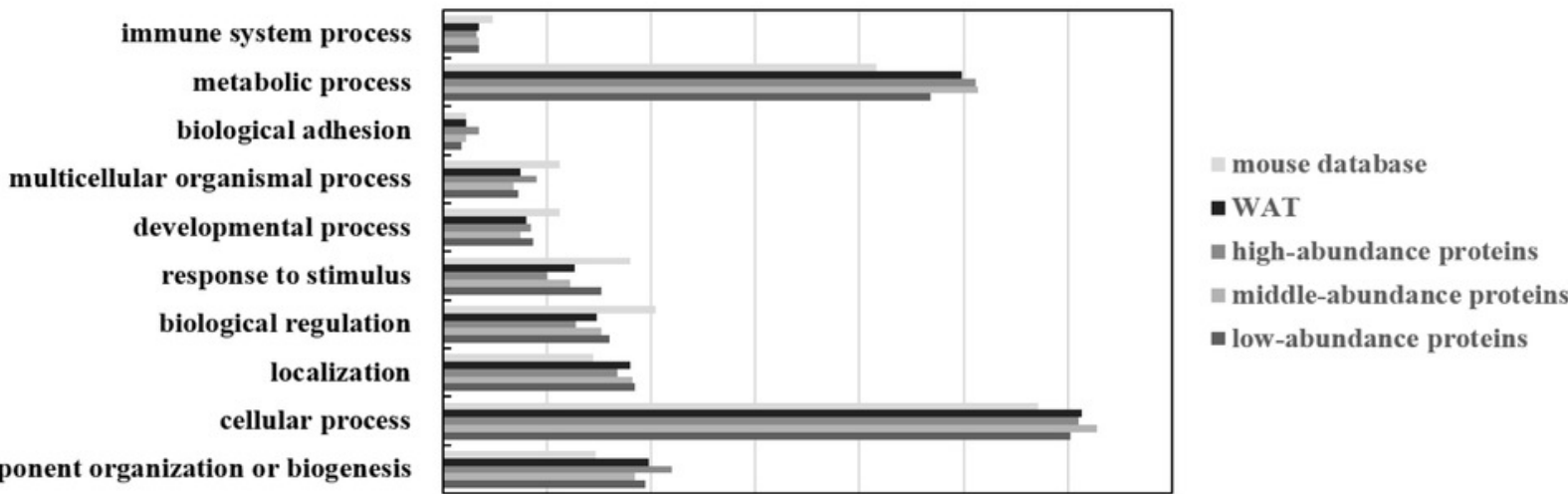

cellular component organization or biogenesis

D

\section{Canonical pathway of WAT}

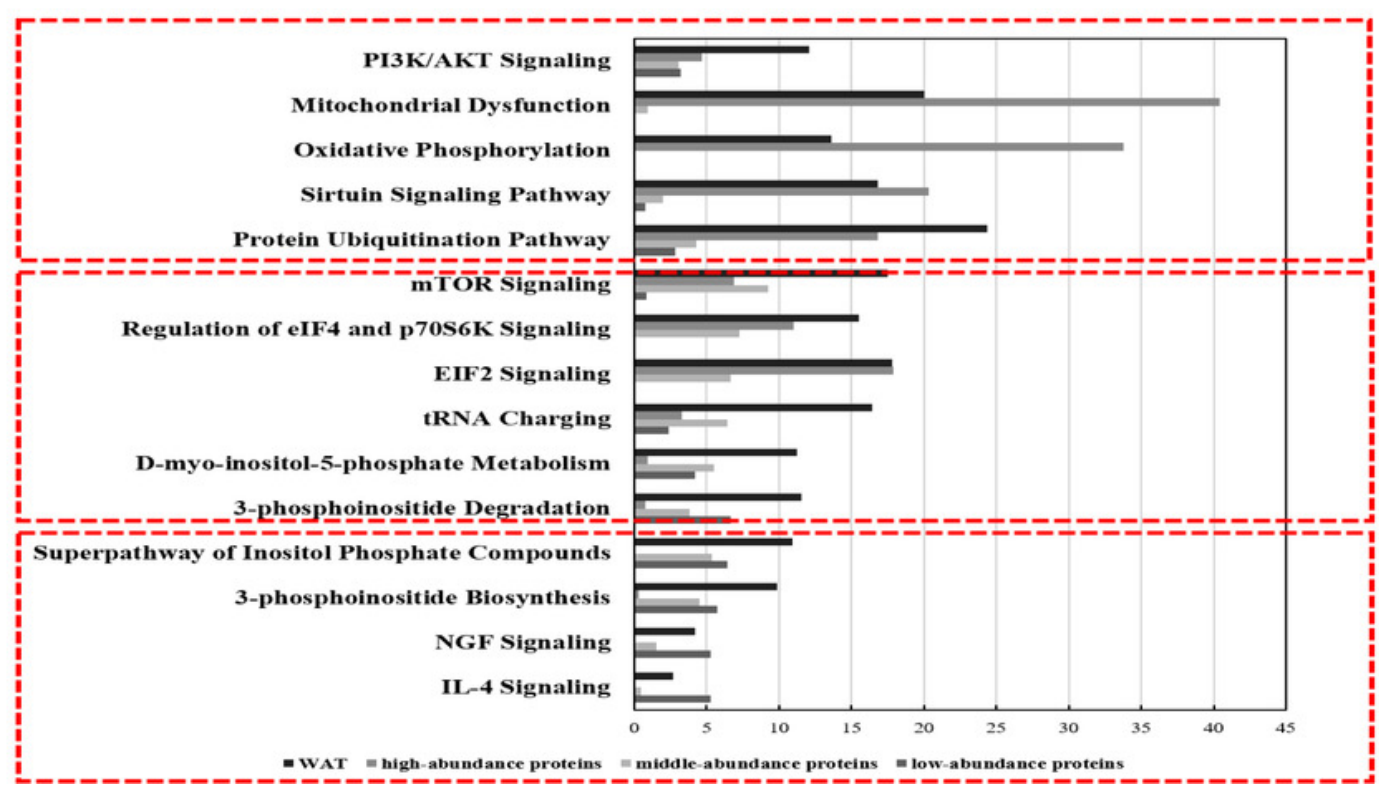

Pathway enrichment in high-abundance proteins

Pathway enrichment in middle-abundance proteins

Pathway enrichment in low-abundance proteins 